TRANSACTIONS OF THE

AMERICAN MATHEMATICAL SOCIETY

Volume 351, Number 7, Pages 2875-2897

S 0002-9947(99)02023-1

Article electronically published on March 8, 1999

\title{
ON THE ENHANCEMENT OF DIFFUSION BY CHAOS, ESCAPE RATES AND STOCHASTIC INSTABILITY
}

\author{
PIERRE COLLET, SERVET MARTÍNEZ, AND BERNARD SCHMITT
}

\begin{abstract}
We consider stochastic perturbations of expanding maps of the interval where the noise can project the trajectory outside the interval. We estimate the escape rate as a function of the amplitude of the noise and compare it with the purely diffusive case. This is done under a technical hypothesis which corresponds to stability of the absolutely continuous invariant measure against small perturbations of the map. We also discuss in detail a case of instability and show how stability can be recovered by considering another invariant measure.
\end{abstract}

\section{INTRODUCTION}

It has been stated several times in the Physics literature that "diffusion enhances chaos". The purpose of this paper is to investigate quantitatively this question for a family of one dimensional dynamical systems (piecewise expanding maps of the interval) with small stochastic perturbations. In the presence of noise the trajectory may jump outside the interval. We will compare the typical time of occurrence of this event for the case of a pure random walk and of a chaotic dynamics stochastically perturbed.

The effect of stochastic perturbations on the long time behavior of some chaotic dynamical systems has received a lot of attention in the past few years (see $[\mathrm{FW}]$ ) but we will be interested here in a different problem connected with the decay of the total probability. This problem can be formulated generally as follows. Start with a map $\Phi$ of a phase space $\Omega$ which has an ergodic invariant measure $\mu$. In other words, one is interested by the sequence of points in the phase space recursively generated by

$$
x_{n+1}=\Phi\left(x_{n}\right) .
$$

Assume now this deterministic process is perturbed by a small stochastic fluctuation occurring every time step. If the stochastic perturbation maps $\Omega$ into itself, the total probability will be conserved. However, if there is a nonzero probability that the small stochastic fluctuation maps the point outside of phase space, the total probability will decrease. Mapping outside the phase space is not a well defined concept at this point but one can imagine for example that $\Omega$ is a subspace of a larger set $\tilde{\Omega}$ such that on the set $\tilde{\Omega} \backslash \Omega$, $\Phi$ and the stochastic map reduce to the identity.

Received by the editors May 1, 1996 and, in revised form, January 23, 1997.

1991 Mathematics Subject Classification. Primary 58F11, 60J99.

(C)1999 American Mathematical Society 
In physical terms, the problem we have in mind is to consider a large assembly of identical independent particles evolving in a box $\Omega$ according to the deterministic map $\Phi$ and small stochastic perturbations. If a particle escapes the box, it will never come back again (it dies!). This is what we call leaking. A natural quantity associated to this problem is the rate of decay of the number of particles in the box for different type of maps $\Phi$ (escape rate). As mentioned above, in order to investigate the influence of chaos on this escape rate we will consider two types of maps of the unit interval: the identity map and regular expanding maps. In both cases we will estimate the rate of escape (of decay of the total probability) when small stochastic perturbations are superposed to these maps. We will see that for small stochastic perturbations, the rate of escape for the stochastic map is much larger for the chaotic map than for the identity map.

A simple argument can be given as follows. If one considers a pure random walk with steps of size $\epsilon>0$, it follows intuitively from the central limit theorem that most particle will need a time of order $\epsilon^{-2}$ to travel a distance of order one needed to leave the interval. On the other hand, if we have a chaotic map with a stochastic perturbation of size $\epsilon$, using Birkhoff's ergodic theorem (see [CG] for a rigorous analysis without noise) it appears that a typical trajectory will need a time of order only $\epsilon^{-1}$ to reach a neighborhood of size $\epsilon$ of the boundary where it has a sizable probability to jump outside. We will prove indeed in Theorems I and II that the above intuitive results are correct.

Our simple model above can be seen as an oversimplification of the Poincaré sections of two dimensional flows, one leading to a completely integrable two dimensional map, the other one to a chaotic dynamic. We also refer to [BWZ] for other arrangements of dynamical systems and physical applications.

In section II we will formulate our hypotheses on the dynamical systems and the stochastic perturbations, and describe the main results about the typical escape times. These results will be proven in section III using techniques developed previously in $[\mathrm{CG}]$ for controlling some (large) perturbations of transfer operators.

A basic hypothesis for controlling perturbations of piecewise expanding maps of the interval is that small invariant segments cannot occur (see [Ke], [BY], [BK], $[\mathrm{BKS}]$ ). In section IV we will consider such a situation where the basic stochastic stability question seems to fail. However, in the presence of an invariant segment absorbing asymptotically all the probability it is natural to study the trajectories which will never penetrate into this segment. Using techniques similar to those developed in section III we prove in a simple case that the trajectories are asymptotically distributed according to an invariant measure supported by a Cantor set, and it is this measure which in the limit converges to the SRB measure of the initial unperturbed map. In other words, in the presence of a small invariant segment produced by perturbation of a mixing map, it is a singular invariant measure which converges (weakly) to the SRB measure of the unperturbed map (when the perturbation goes to zero) and not the SRB measure of the perturbed map. Although we prove the result here in a special case, it is easy to extend our method to a larger class of situations and we conjecture that a similar phenomenon occurs whenever small invariant segments are produced by perturbations of mixing piecewise expanding maps. When the trajectories can exit from the phase space, instead of looking for stationary distributions one should look for quasi-invariant distributions as those described in this work. When the phase space is not compact their existence as well as the study of the process involved is nontrivial. In this context, 
see [FKMP] for the study of quasi-invariant distributions for Markov chains and [CMSM] for diffusions. We recall that if $\left(X_{t}\right)$ is a Markov process and $\tau$ is the first exit time of some Borel subset $I$ of the phase space, then a probability measure $\mu$ is a quasi-invariant distribution if $\mathbb{P}_{\mu}\left\{X_{t} \in C \mid \tau>t\right\}=\mu$ for all Borel subsets $C \subset I$.

\section{ESCAPE RATES FOR CHAOS AND DIFFUSION}

We will denote by $\mathcal{B}$ the vector space of functions with bounded variation on the interval $[0,1]$. We will sometimes implicitly identify this class of functions with their extensions vanishing outside $[0,1]$. This space will be equipped with different (equivalent) norms which depend on a parameter $\theta>0$. These norms are given by

$$
\|g\|_{\theta}=\theta \vee g+\|g\|_{1}
$$

where \|\|$_{p}$ is the $L^{p}$ norm with respect to the Lebesgue measure, and $\vee g$ is the variation of $g$. It is easy to verify that equipped with any of these norms, the space $\mathcal{B}$ becomes a Banach space denoted below by $\mathcal{B}_{\theta}$. For simplicity we will denote by $\|\mid\|$ the norm corresponding to $\theta=1$, and again by $\mathcal{B}$ the associated Banach space. Notice that all the norms $\left|\|\mid\|_{\theta}\right.$ are equivalent.

We will denote by $f$ a piecewise regular expanding map of the interval $[0,1]$. This is a map which satisfies the following hypotheses.

Hypothesis H1. i) There is a finite increasing sequence of $l+1$ points $a_{0}=0<$ $a_{1}<\cdots<a_{l-1}<a_{l}=1$ such that on each interval $] a_{i}, a_{i+1}[$ the function $f$ is regular $\left(C^{2}\right)$ and can be extended by continuity to a regular $\left(C^{2}\right)$ function of the closed interval.

ii) There is a number $A>0$ and a number $\rho>1$ such that $\forall n$

$$
\inf _{x}\left|f^{n^{\prime}}(x)\right| \geq A \rho^{n} .
$$

It is well known (see $[\mathrm{LY}],[\mathrm{C}]$ ) that such maps admit an absolutely continuous invariant measure. There is also a finite decomposition into mixing components which are also regular expanding maps of some interval, and we shall assume below that one of these components has been selected.

Hypothesis H2. The map $f$ has a unique absolutely continuous invariant measure (a.c.i.m.) $h_{0} d x$ which is ergodic and mixing.

The basic tool to establish the above results is the so-called transfer operator defined for a map $f$ satisfying $\mathbf{H} \mathbf{1}$ by its action on $\mathcal{B}$,

$$
P g(x)=\sum_{y: f(y)=x} \frac{g(y)}{\left|f^{\prime}(y)\right|} .
$$

Since the domain of $f$ is the interval all preimages $y$ belong to $[0,1]$. Below several maps will be considered and when there is some ambiguity we will denote by $P_{f}$ the transfer operator of the map $f$.

The densities of the absolutely continuous invariant measures are the (nonnegative) eigenvectors of $P$ of eigenvalue 1 . Under hypothesis $\mathbf{H 1}$, it is known ([LY]) that $P$ is a bounded linear operator in $\mathcal{B}$. Moreover, there is a fundamental estimate of Lasota and Yorke ([LY]) stating that there is a number $\tilde{\alpha}<1$, and a number $C>1$ such that for any function $g \in \mathcal{B}$ and for any integer $n \geq 0$ we have

$$
\vee\left(P^{n} g\right) \leq C\left(\tilde{\alpha}^{n} \vee g+\|g\|_{1}\right)
$$


Under the stronger hypothesis H2, the peripheral spectrum of $P$ consists of the only simple eigenvalue 1 , with a nonnegative eigenvector denoted by $h_{0}$ and eigenform $e_{0}$ which is the integration against the (normalized) Lebesgue measure of the interval. We shall also assume that $h_{0}$ is of integral 1 . The rest of the spectrum is contained in a disk of radius strictly smaller than one, and from the spectral decomposition Theorem (see $[\mathrm{HK}]$ ) one concludes that there exists a nonnegative number $r_{0}<1$, a positive constant $B$ and an operator $R$ commuting with $P$ such that

$$
P^{n}=h_{0} e_{0}+R^{n} \quad \text { with for any integer } n \quad\left\|R^{n} \mid\right\| \leq B r_{0}^{n} .
$$

$h_{0}$ is the density of the absolutely continuous invariant measure.

We now describe the stochastic perturbations of the map $f$. A simple example would be to consider a sequence of independent identically distributed random variables $\left(\xi_{n}\right)$ with values in the interval $[-1,1]$. For a positive number $\epsilon$ we can now define recursively a Markov process $\left(X_{n}\right)$ by $X_{0}=x$ and

$$
X_{n+1}=f\left(X_{n}\right)+\epsilon \xi_{n} .
$$

We will denote below by $f_{\xi}$ this random map, namely

$$
f_{\xi}(x)=f(x)+\epsilon \xi .
$$

One can extend the results below to more general cases of random maps. We will, however, explain the results and give complete proofs only for the above type of random perturbations, with the i.i.d. random variables $\left(\xi_{n}\right)$ having a probability density $\varphi$ supported by $[-1,1]$, even and $C^{1}$. This insures that the average of $\varphi$ is zero and then there is no drift in the noise. On the other hand, compactness of the support of $\varphi$ is assumed for simplicity, but most of the results can be proven with less stringent assumptions.

Of course, if some $f\left(X_{p}\right)$ happens to be at a distance less than $\epsilon$ of the boundary ( 0 or 1$)$, there is a nonzero probability that $X_{p+1}$ will be outside the interval $[0,1]$ where the map $f$ is undefined. Associated to the stochastic perturbation, there is also a transfer operator $\mathcal{U}$ given by

$$
\mathcal{U} g(x)=\mathbb{E}\left(P_{f_{\xi}} g(x)\right)=\int \sum_{y: f_{\xi}(y)=x} \frac{g(y)}{\left|f_{\xi}^{\prime}(y)\right|} \varphi(\xi) d \xi .
$$

It is easy to verify that if a random variable $X$ with values in $[0,1]$ has a density $g$ with respect to the Lebesgue measure, then if $\xi$ is independent of $X$, the random variable $f_{\xi}(X)$ has a density $\mathcal{U} g$ with respect to the Lebesgue measure. If we ask about the probability that $f_{\xi}(X)$ is also in $[0,1]$, it is given by

$$
\int_{0}^{1} \mathcal{U} g(x) d x
$$

More generally, if $X_{0}$ has distribution with density $g$ supported by the unit interval, we have for any integer $n \geq 0$

$$
\mathbb{P}\left\{X_{j} \in[0,1], j=0, \cdots, n\right\}=\int_{0}^{1} Q^{n} g(x) d x,
$$

where

$$
Q g(x)=\chi_{[0,1]}(x) \mathcal{U} g(x)
$$


and $\chi_{A}$ denotes the characteristic function of the set $A$. Note that $Q$ can be written as $Q=\tilde{Q} P$ where $\tilde{Q}$ is the operator given by

$$
\tilde{Q} g(x)=\chi_{[0,1]}(x) \mathbb{E}_{\xi}\left(\chi_{[0,1]}(x-\epsilon \xi) g(x-\epsilon \xi)\right) .
$$

One of the goals of this paper is to study the behavior of the sequence $\mathbb{P}\left\{X_{j} \in\right.$ $[0,1], j=0, \cdots, n\}$ for large $n$ and small $\varepsilon$. We will see that for $\epsilon$ small enough, this sequence decays exponentially fast with $n$. In the particle interpretation given in the introduction, this means that the number of particles which stay in the interval up to time $n$ decays exponentially fast. We will establish below (under adequate hypotheses on the stochastic perturbation) that this decay rate is $-1 / \log \lambda$ where $\lambda$ is the largest eigenvalue of $Q$.

Another interesting question is about the distribution of the remaining particles. Namely we condition the process $\left(X_{n}\right)$ to stay in the interval, and we ask for the asymptotic law of this process. We will see below that asymptotically, under the condition that the process stayed in $[0,1]$ up to time $n$, the random variable $X_{n}$ is distributed according to the normalized quasi-invariant measure corresponding to the eigenvalue $\lambda$.

These results will be proven using perturbation theory between $P$ and $Q$. Intuitively, if $\epsilon$ is small, one expects $P-Q$ to be small. However, it turns out that this is not so in $\mathcal{B}$ because by stochastic perturbations the intervals of discontinuities of the map can move. As a consequence, in the variation norm, $P-Q$ may be of order one. This problem is solved below by using several adequate values $\theta$ in the norm $\mid\|\quad\| \|_{\theta}$ to balance more optimally the size of the perturbation terms. The technique of introducing several norms to balance seemingly nonsmall terms in perturbation theory of Ruelle Perron Frobenius operators was introduced in [CG] (see also [C]). It has since been used to prove several results: [BY], [BIS].

We now make an important hypothesis on the orbits of the points where $f$ is not regular, that is to say the points $\left(a_{j}\right)$, which will ensure the stability of the stochastic perturbation. It is known (see $[\mathrm{Ke}],[\mathrm{BY}]$ ) that if this condition is not satisfied, one may observe stochastic instability. In particular we want to prevent the occurrence of small invariant segments by small perturbations of $f$. We refer to $[\mathrm{Ke}],[\mathrm{BY}],[\mathrm{BK}]$ for similar hypotheses. In Section IV we will give a detailed analysis of a typical example, resolving this (apparent) instability.

\section{Hypothesis H3.}

(1) Any periodic point $a_{j}$ from the definition of $f$ is either a point of discontinuity or $f^{\prime}\left(a_{j}^{-}\right) f^{\prime}\left(a_{j}^{+}\right)>0$.

(2) For any integer $m \geq 0$, we have

$$
f^{m+2}(\partial \mathcal{A}) \cap\{0,1\}=\emptyset
$$

where $\partial \mathcal{A}=\left\{a_{0}, \ldots, a_{\ell}\right\}$.

A similar hypothesis was also introduced in [CG]. We observe that 2) is not satisfied for the simple map $2 x(\bmod 1)$. In fact 2$)$ is not really needed in that case, one can still prove the theorem below at the cost of using more detailed estimates.

Theorem 1. Assume hypotheses H1-H3 are satisfied and $\varphi$ even and $C^{1}$, supported in $[-1,1]$. Then for $\epsilon<1$ small enough, there is a number $a>0$ given 
by

$$
a=\left[-h_{0}\left(0_{+}\right) \int_{-1}^{0} \xi \varphi(\xi) d \xi+h_{0}\left(1_{-}\right) \int_{0}^{1} \xi \varphi(\xi) d \xi\right]
$$

such that the operator $Q$ has a peripheral spectrum consisting of a unique positive simple eigenvalue $\lambda(\epsilon)$ which satisfies the estimate

$$
\lambda(\epsilon)=1-a \epsilon+o(\epsilon) .
$$

The associated nonnegative (integrable) eigenvector $h_{\epsilon}$ normalized by the condition

$$
\int h_{\epsilon} d x=1
$$

is the density of an absolutely continuous quasi-invariant distribution with respect to the time of first exit of the process $\left(X_{n}\right)$ from the interval $[0,1]$. Moreover, if we denote by $\tau$ this exit time then the following limit exists

$$
\lim _{n \rightarrow \infty} \mathbb{P}_{x}\left\{X_{1} \in A_{1}, \cdots, X_{k} \in A_{k} \mid \tau>n\right\}=\tilde{\mathbb{P}}_{x}\left\{X_{1} \in A_{1}, \cdots, X_{k} \in A_{k}\right\},
$$

and defines a law on trajectories which never leave the interval. $\tilde{\mathbb{P}}$ is given by the Markov kernel $M$ defined on $[0,1]$ by

$$
M(x, d y)=\lambda(\epsilon)^{-1} \frac{h_{\epsilon}(y)}{h_{\epsilon}(x)} \varphi\left(\frac{y-f(x)}{\epsilon}\right) \frac{d y}{\epsilon} .
$$

The eigenform $e_{\epsilon}$ corresponding to $\lambda(\epsilon)$ is a positive measure, the probability measure $h_{\epsilon} d e_{\epsilon}$ is invariant for the kernel $M$.

As explained in the introduction, we want to compare the above result to a situation where there is no deterministic chaotic dynamic but essentially an integrable one. For simplicity we shall take for $f$ the case of the identity map, and define the associated stochastic perturbation by simply adding independent noises, namely

$$
f_{\xi}(x)=x+\epsilon \xi \text {. }
$$

The iteration generates of course a random walk with independent increments distributed as $\xi$. The transfer operator is simply given by $\tilde{Q}$.

Theorem 2. There is a number $\epsilon_{d}>0$ such that for $\left.\left.\epsilon \in\right] 0, \epsilon_{d}\right]$ the operator $\tilde{Q}$ has a peripheral spectrum consisting of a unique simple eigenvalue $\lambda_{d}(\epsilon)>0$ satisfying

$$
1>\lambda_{d}(\epsilon)>1-\frac{\pi^{2} m_{2}}{2} \epsilon^{2}+\mathcal{O}(1) \epsilon^{3},
$$

where $m_{2}$ is the second moment of the random variable $\xi$,

$$
m_{2}=\int_{-1}^{1} \xi^{2} \varphi(\xi) d \xi
$$

We conjecture that $\lambda_{d}(\epsilon)=1-\frac{\pi^{2} m_{2}}{2} \epsilon^{2}+\mathcal{O}(1) \epsilon^{3}$. The difficulty in estimating the spectrum of $\tilde{Q}$ is that when $\epsilon$ tends to zero, this operator formally converges to the identity which has an infinitely degenerate eigenvalue 1.

As explained in the introduction, the above two results provide a quantitative difference between the escape rates in the two cases with and without chaos. 


\section{Proofs of Theorems I AND II}

Proposition 3. Under condition $\mathbf{H 1}$, and $\mathbf{H 3}(\mathbf{1})$ there are numbers $1>\alpha>\tilde{\alpha}$, $\beta>C>1$, and $1>\epsilon_{0}>0$ such that for any $\epsilon \in\left[0, \epsilon_{0}\right]$, for any function $g \in \mathcal{B}$ and any integer $n \geq 0$ we have

$$
\begin{gathered}
\vee\left(\left(Q^{n}-P^{n}\right) g\right) \leq \beta \alpha^{n} \vee g+\beta\left\|_{1}\right\|_{1} \\
\int|Q g-P g| d x \leq \beta \epsilon\left(\vee g+\|g\|_{1}\right) \quad \text { and also } \quad\|Q\|_{1} \leq 1 .
\end{gathered}
$$

Proof. The first estimate (2) follows from the (LY) estimate and from the bound

$$
\vee\left(Q^{n} g\right) \leq \beta_{1} \alpha_{1}^{n} \vee g+\beta_{1}\|g\|_{1},
$$

where $0<\alpha_{1}<1$ and $\beta_{1}>0$. The proof of this estimate requires only minor modifications from the proof of a similar result in [BY]. We leave these modifications to the reader.

We now prove the second part of Proposition 3. We will first prove that

$$
\|(\tilde{Q}-I) g\|_{1} \leq \mathcal{O}(1) \epsilon\left(\vee g+\|g\|_{1}\right)
$$

from which the first part of (3) follows using the estimate (LY) since $Q-P=$ $(\tilde{Q}-I) P$. We have obviously

$$
\begin{gathered}
\|(\tilde{Q}-I) g\|_{1}=\int_{0}^{\epsilon}|(\tilde{Q}-I) g(x)| d x+\int_{\epsilon}^{1-\epsilon}|(\tilde{Q}-I) g(x)| d x+\int_{1-\epsilon}^{1}|(\tilde{Q}-I) g(x)| d x \\
\leq 4 \epsilon\|g\|_{\infty}+\int_{\epsilon}^{1-\epsilon}|(\tilde{Q}-I) g(x)| d x \leq 4 \epsilon\left(\vee g+\|g\|_{1}\right)+\int_{\epsilon}^{1-\epsilon}|(\tilde{Q}-I) g(x)| d x .
\end{gathered}
$$

Since $g$ is a function of bounded variation, there is a positive measure $\nu$ with total mass $\vee g$ such that if $0 \leq a<b \leq 1$

$$
|g(b)-g(a)| \leq \int_{[a, b]} d \nu .
$$

Using the formula

$$
\tilde{Q} g(x)-g(x)=\chi_{[0,1]}(x) \mathbb{E}_{\xi}(g(x+\epsilon \xi)-g(x))
$$

one obtains

$$
\begin{gathered}
\int_{\epsilon}^{1-\epsilon}|(\tilde{Q}-I) g(x)| d x \leq \int_{\epsilon}^{1-\epsilon} d x \mathbb{E}_{\xi}\left(\int_{\inf (x+\epsilon \xi, x)}^{\sup (x+\epsilon \xi, x)} d \nu\right) \\
=\mathbb{E}_{\xi}\left(\int_{\epsilon}^{1-\epsilon} d x \int_{\inf (x+\epsilon \xi, x)}^{\sup (x+\epsilon \xi, x)} d \nu(y)\right) .
\end{gathered}
$$

Using Fubini's Theorem one gets

$$
\int_{\epsilon}^{1-\epsilon}|(\tilde{Q}-I) g(x)| d x \leq \mathbb{E}_{\xi}\left(\int_{0}^{1} d \nu(y) \int_{\inf (y, y-\epsilon \xi)}^{\sup (y, y-\epsilon \xi)} d x\right) \leq \epsilon \vee g \mathbb{E}(|\xi|) .
$$

This ends the proof of (3). The proof of the last result is elementary and left to the reader. 
Corollary 4. For any $\theta>0$, we have

$$
\begin{aligned}
\left\|Q^{n} g-P^{n} g\right\| \|_{\theta} & \leq \beta \alpha^{n} \theta \vee g+\beta \theta\|g\|_{1}+\frac{\beta^{2} \epsilon}{1-\alpha} \vee g+\beta n \epsilon(1+\beta)\|g\|_{1} \\
& \leq \beta\left[\alpha^{n}+\frac{\beta \epsilon \theta^{-1}}{1-\alpha}+\theta+n \epsilon(1+\beta)\right]\|g\|_{\theta} .
\end{aligned}
$$

This follows at once from the previous result and the definition of the $\theta$ norm. A first step towards a proof of Theorem 1 is the following result.

Proposition 5. There is a number $n_{1}>0$ such that for $\epsilon$ small enough, the operator $Q$ has only a simple positive eigenvalue $\lambda(\epsilon)$ outside the disk of radius $2^{-1 / n_{1}}$. The eigenvector $h_{\epsilon}$ can be chosen nonnegative of integral one. The associated eigenform $e_{\epsilon}$ is a positive measure which satisfies

$$
\left|e_{\epsilon}(1)-1\right| \leq \mathcal{O}(1) \epsilon \log \epsilon^{-1} .
$$

Proof. Let $0<\theta_{0}<1$ be a positive number that we will fix below. By a direct computation we have

$$
\left\|\left|e_{0}\right|\right\|_{\theta_{0}}=1 \text { and }\left\|\left|h_{0}\right|\right\|_{\theta_{0}} \leq(1+C) .
$$

This implies

$$
\text { || } P_{0} \|_{\theta_{0}} \leq(1+C) .
$$

Now, since $P_{0}$ is a projection of rank one, it is easy to compute explicitly its resolvent, namely

$$
R_{\zeta}^{0}=\left(P_{0}-\zeta\right)^{-1}=\frac{P_{0}}{1-\zeta}-\frac{I-P_{0}}{\zeta}
$$

and we get

$$
\sup _{|\zeta|=1 / 2}\left\|\left|R_{\zeta}^{0}\right|\right\|_{\theta_{0}} \leq 6(1+C) .
$$

Now choose for $n$ the smallest integer $n_{1}$ such that

$$
(1+C)^{3}\left[(B+\beta) r_{0}^{n_{1} / 2}+\beta \alpha^{n_{1}}\right]<1 / 36
$$

and then take

$$
\theta_{0}=r_{0}^{n_{1} / 2} .
$$

It follows from our previous estimates that there is a number $0<\epsilon_{1}<\epsilon_{0}<1$ such that for any $\epsilon \in\left[0, \epsilon_{1}[\right.$

$$
\sup _{|\zeta|=1 / 2}\left\|\left|R_{\zeta}^{0}\right|\right\|_{\theta_{0}}\left\|\left|R^{n_{1}}+\left(Q^{n_{1}}-P^{n_{1}}\right) \|\right|_{\theta_{0}}<1 .\right.
$$

From now on, we will always assume that $\epsilon$ is smaller than $\epsilon_{1}$. Since $Q^{n_{1}}=$ $P_{0}+R^{n_{1}}+\left(Q^{n_{1}}-P^{n_{1}}\right)$, by perturbation theory (see [K, II.§3]) we deduce that $Q^{n_{1}}$ has a unique simple eigenvalue $\gamma(\epsilon)$ outside $D_{1 / 2}$ and the rest of the spectrum is contained inside that disk. It follows easily from (4) that

$$
\left\|\int_{|\zeta|=1 / 2} R_{\zeta}^{0}\left(R^{n_{1}}+Q^{n_{1}}-P^{n_{1}}\right) R_{\zeta} d \zeta\right\|_{\theta_{0}}<\frac{1}{1+C},
$$


where $R_{\zeta}$ denotes the resolvent of $Q^{n_{1}}$. Let

$$
\hat{h}_{\epsilon}=\left(I-\int_{|\zeta|=\frac{1}{2}} R_{\zeta} d \zeta\right) h_{0},
$$

from the resolvent equation it follows that

$$
\hat{h}_{\epsilon}=h_{0}+\int_{|\zeta|=\frac{1}{2}} R_{\zeta}^{0}\left(R^{n_{1}}+Q^{n_{1}}-P^{n_{1}}\right) R_{\zeta} h_{0} d \zeta .
$$

From our previous estimates and $\epsilon>0$ small enough we find $e_{0}\left(\hat{h}_{\epsilon}\right)>0$. Therefore, the eigenvector $h_{\epsilon}$ normalized by $e_{0}\left(h_{\epsilon}\right)=1$ (namely $h_{\epsilon}=\left(e_{0}\left(\hat{h}_{\epsilon}\right)\right)^{-1} \hat{h}_{\epsilon}$ ) satisfies

$$
\left\|\left|h_{\epsilon}\right|\right\|_{\theta_{0}} \leq \mathcal{O}(1)
$$

uniformly in $\epsilon$, and by equivalence of norms,

$$
\left\|\left|h_{\epsilon} \|\right| \leq \mathcal{O}(1) \theta_{0}^{-1}\right.
$$

also uniformly in $\epsilon$ small enough

The spectral properties for $Q$ follow immediately. Indeed, we conclude that $Q$ has a simple eigenvalue $\lambda(\epsilon)$ outside of the disk of radius $2^{-n_{1}}$ with eigenvector $h_{\epsilon}$. $\lambda(\epsilon)$ must be one of the complex $n_{1}$ roots of $\gamma(\epsilon)$ but since $Q$ is a positivity preserving operator, $\lambda(\epsilon)$ must be the only positive one (since $Q^{n_{1}}$ is a positivity preserving operator, $\gamma(\epsilon)$ is real and positive).

We now prove the estimate on $e_{\epsilon}(1)$. From the spectral property of $Q$ and perturbation theory we have for any integer $N$

$$
Q^{N}=h_{\epsilon} \lambda(\epsilon)^{N} e_{\epsilon}+T^{N},
$$

where one can choose $\epsilon_{1}>0$ small enough such that there are two constants $C_{1}>0$ and $r_{2} \in\left[0,1[\right.$ such that for any $\left.\epsilon \in] 0, \epsilon_{1}\right]$ we have

$$
\left\|T^{N} \mid\right\| \leq C_{1} r_{2}^{N} .
$$

This implies (since $e_{0}\left(h_{\epsilon}\right)=1$ )

$$
1+e_{0}\left(\left(Q^{N}-P^{N}\right) 1\right)=e_{0}\left(Q^{N} 1\right)=\lambda(\epsilon)^{N} e_{\epsilon}(1)+\mathcal{O}\left(r_{2}^{N}\right) .
$$

On the other hand we have

$$
\begin{aligned}
e_{0}\left(\left(Q^{N}-P^{N}\right) 1\right) & =\sum_{j=0}^{N-1} e_{0}\left(P^{j}(Q-P) Q^{N-j-1} 1\right) \\
& \left.=\sum_{j=0}^{N-1} e_{0}(Q-P) Q^{N-j-1} 1\right)=\epsilon N \mathcal{O}(1)
\end{aligned}
$$

by Proposition 3 and (LY). In other words, we have

$$
\left|\lambda(\epsilon)^{N} e_{\epsilon}(1)-1\right| \leq \mathcal{O}(1)\left(\epsilon N+r_{2}^{N}\right) .
$$

We take $N_{0}=\left[\log \epsilon^{-1}\right]$ and denote $\chi=\lambda(\epsilon)^{N_{0}}$, so the last equation applied with $N=N_{0}$ and $N=2 N_{0}$ gives $\left|\chi e_{\epsilon}(1)-1\right| \leq \eta_{1},\left|\chi^{2} e_{\epsilon}(1)-1\right| \leq \eta_{2}$ with $\eta_{1}$ and $\eta_{2}$ or order $\mathcal{O}(1) \epsilon \log \epsilon^{-1}$. The second inequality reads

$$
1+\eta_{2}>\chi(\chi a)>1-\eta_{2}
$$


and for $\epsilon$ small enough combining with the first inequality we get

$$
\frac{1+\eta_{2}}{1-\eta_{1}}>\chi>\frac{1-\eta_{2}}{1+\eta_{1}}
$$

and finally by using again the first inequality we obtain

$$
\frac{\left(1+\eta_{1}\right)^{2}}{1-\eta_{2}}>e_{\epsilon}(1)>\frac{\left(1-\eta_{1}\right)^{2}}{1+\eta_{2}} .
$$

By taking into account that $\eta_{i} \sim \mathcal{O}(1) \epsilon \log \epsilon^{-1}$ for $i=1,2$ we find the result.

We now prove some intermediate results that will allow us to improve the conclusions of Proposition 3.

Lemma 6. If $\varphi$ has compact support in the interval $[-1,1]$, there is (for $\epsilon$ small enough) a nonnegative function $\omega_{\epsilon}$ with support in $[0, \epsilon] \cup[1-\epsilon, 1]$ bounded by one and given by

$$
\omega_{\epsilon}(x)=\chi_{[0,1]}(x) \int_{-1}^{1} \varphi(\xi) d \xi\left(1-\chi_{[0,1]}(x+\epsilon \xi)\right)
$$

such that for any integrable function $g$ with support in $[0,1]$ we have

$$
e_{0}(\tilde{Q}-I) g=-\int_{0}^{1} \omega_{\epsilon}(x) g(x) d x
$$

Moreover,

$$
e_{0}(\tilde{Q}-I) h_{0}=-\epsilon\left[-h_{0}\left(0_{+}\right) \int_{-1}^{0} \xi \varphi(\xi) d \xi+h_{0}\left(1_{-}\right) \int_{0}^{1} \xi \varphi(\xi) d \xi\right]+o(\epsilon) .
$$

Proof. One easily gets by a linear change of variable the expression for $\omega_{\epsilon}(x)$ from which the first part of the lemma follows immediately. The second part follows since a function of bounded variation has a left and a right limit.

We will denote below by $\sigma$ the number (larger than 1 )

$$
\sigma=\sup _{x}\left|f^{\prime}(x)\right|
$$

and by $d$ we denote the usual distance on the interval.

Lemma 7. There is a constant $K_{1}>0$ such that for any integer $n>0$ and for any $1>\epsilon>0$ satisfying $3(1+\sigma)^{n} \epsilon \leq \delta_{0}=\inf _{0 \leq j<l}\left|a_{j+1}-a_{j}\right|$ we have

$$
\sup _{\left\{x: d\left(x, \bigcup_{j=1}^{n} f^{j}(\partial \mathcal{A})\right)>\epsilon(1+\sigma)^{n}\right\}}\left|\left(Q^{n} 1\right)^{\prime}(x)\right| \leq K_{1}^{n} .
$$

Proof. The proof is recursive. We first define three positive constants by

$$
C_{1}=\sup _{x}\left|f^{\prime}(x)\right|^{-2}, C_{2}=\sup _{x}\left|f^{\prime \prime}(x)\right| /\left|f^{\prime}(x)\right|^{3}, C_{3}=\sup _{x, n} Q^{n} 1(x)+1 .
$$

The last constant is finite because the nonnegative function $Q^{n} 1$ has an integral bounded by one and a uniformly bounded variation by Proposition 1 .

It is useful to introduce the set $V_{n}$ defined by

$$
V_{n}=\left\{x: d\left(x, \bigcup_{j=1}^{n} f^{j}(\partial \mathcal{A})\right) \leq \epsilon(1+\sigma)^{n}\right\},
$$


We have

$$
Q g(x)=\mathbb{E}_{\xi}\left\{\frac{\sum_{I \in \mathcal{A}_{\xi}} g\left(\psi_{I}(x, \xi)\right) \chi_{f_{\xi}(I)}(x)}{\left|f^{\prime}\left(\psi_{I}(x, \xi)\right)\right|}\right\},
$$

where $\mathcal{A}_{\xi}$ denotes the set of subintervals $I \subset[0,1]$, where $f_{\xi}$ is monotone, regular and maps $I$ into $[0,1]$, and $\psi_{I}(x, \xi)$ denotes the inverse of $f_{\xi}$ from $f_{\xi}(I)$ to $I$. Observe that for $\epsilon$ small enough, $\operatorname{card}\left(\mathcal{A}_{\xi}\right)=l$.

Note that from the definition of $f_{\xi}$ any point in the boundary of an interval $f_{\xi}(I)$ with $I \in \mathcal{A}_{\xi}$ is at a distance at most $\epsilon$ of $f(\partial \mathcal{A})$. From the previous formula it is then immediate to verify that for $x \notin V_{1}$ we have

$$
\left|Q 1^{\prime}(x)\right| \leq l C_{2}<K_{1}
$$

if $K_{1}>l C_{2}$. We now assume that the Lemma has been proven up to an integer $n>1$. We define

$$
\tilde{V}_{n+1}=\left\{x: \psi_{I}(x, \xi) \in V_{n} \text { for some } \xi \text { with }|\xi| \leq 1 \text { and some } I \in \mathcal{A}_{\xi}\right\} \cup V_{1} .
$$

Since $Q^{n} 1$ is differentiable on the complement of $V_{n}$, it follows easily that $Q^{n+1} 1$ is differentiable on the complement of $\tilde{V}_{n+1}$. Moreover, using the bound on the derivative of $Q^{n} 1$ and the explicit expression (6) for $Q$ we have since $f^{\prime}=f_{\xi}^{\prime}$

$$
\sup _{x \notin \tilde{V}_{n+1}}\left|\left(Q^{n+1} 1\right)^{\prime}(x)\right| \leq l\left(C_{2} C_{3}+C_{1} K_{1}^{n}\right) \leq K_{1}^{n+1}
$$

if $K_{1}=l\left(C_{2} C_{3}+C_{1}\right)+1$.

We finally check that $\tilde{V}_{n+1} \subset V_{n+1}$. First of all, by definition, $V_{1} \subset V_{n+1}$. We now observe that if $\psi_{I}(x, \xi) \in V_{n}$, then

$$
x \in f_{\xi}\left(V_{n} \cap \bigcup_{I \in \mathcal{A}_{\xi}} I\right) .
$$

We also observe that $V_{n}$ is a finite union of intervals $J$ of width at most $2 \epsilon(1+\sigma)^{n}$ each centered at a point of $\bigcup_{j=1}^{n} f^{j}(\partial \mathcal{A})$. There are now two cases. Either $f_{\xi}$ is differentiable on $J$, in which case $f_{\xi}(J)$ is an interval containing a point $\zeta$ of $\bigcup_{j=2}^{n+1} f^{j}(\partial \mathcal{A})+\epsilon \xi$ and each point of $f_{\xi}(J)$ is at a distance less than $\epsilon+\epsilon \sigma(1+\sigma)^{n}$ of $\zeta$. Therefore, $f_{\xi}(J)$ is contained in $V_{n+1}$. In the other case, since $\epsilon(1+\sigma)^{n}<\delta_{0} / 2$, $f_{\xi}(J)$ is the union of at most two intervals containing a point of $\bigcup_{j=1}^{n+1} f^{j}(\partial \mathcal{A})+\epsilon \xi$. It follows as before that these two intervals are also in $V_{n+1}$.

We remark that using better estimates on the derivatives, in the above lemma one can replace the factor $K_{1}^{n}$ by a constant uniform in $n$.

Corollary 8. There is a constant $K>0$ such that for any integer $n>0$ and for any $\epsilon>0$ satisfying $3(1+\sigma)^{n} \epsilon \leq \delta_{0}=\inf _{0 \leq j<l}\left|a_{j+1}-a_{j}\right|$ we have

$$
\sup _{\left\{x: d\left(x, \cup_{j=1}^{n} f^{j}(\partial \mathcal{A})\right)>\epsilon(1+\sigma)^{n}\right\}}\left|\left(P Q^{n-1} 1\right)^{\prime}(x)\right| \leq K^{n} .
$$

The proof is similar to the proof of the previous lemma and is left to the reader. 
Proof of Theorem 1. Let $N$ denote a large integer (but of order $o\left(\log \epsilon^{-1}\right)$ ) depending on $\epsilon$ to be fixed later on. We first have easily since $e_{0}\left(h_{\epsilon}\right)=1$

$$
\begin{aligned}
\lambda-1= & e_{0}\left((Q-P) h_{\epsilon}\right)=\lambda^{-N} e_{0}\left((Q-P) Q^{N} h_{\epsilon}\right) \\
= & \lambda^{-N} e_{0}\left((Q-P) P^{N} h_{\epsilon}\right)+\lambda^{-N} e_{0}\left((Q-P)\left(Q^{N}-P^{N}\right) h_{\epsilon}\right) \\
= & \lambda^{-N} e_{0}\left((Q-P) h_{0}\right)+\lambda^{-N} e_{0}\left((Q-P)\left(P^{N} h_{\epsilon}-h_{0}\right)\right) \\
& +\sum_{j=0}^{N-1} \lambda^{-j-1} e_{0}\left((Q-P) P^{j}(Q-P) h_{\epsilon}\right) .
\end{aligned}
$$

From the first equality and Lemma 6 we get

$$
\lambda-1=e_{0}\left((\tilde{Q}-I) P h_{\epsilon}\right)=\int_{0}^{1} \omega_{\epsilon}(x) P h_{\epsilon}(x) d x=\mathcal{O}(\epsilon) .
$$

We now observe using again $e_{0}\left(h_{\epsilon}\right)=1$ and equation (1) that $P^{N+1} h_{\epsilon}-h_{0}=v$ with

$$
\vee v+\|v\|_{1} \leq B r_{0}^{N+1}\left(\vee h_{\epsilon}+\left\|h_{\epsilon}\right\|_{1}\right) \leq \mathcal{O}(1) r_{0}^{N},
$$

since $\theta_{0}^{-1}=\mathcal{O}(1)$ and (5).

This implies using Lemma 6 that

$\left|e_{0}(Q-P)\left(P^{N} h_{\epsilon}-h_{0}\right)\right|=\left|e_{0}(\tilde{Q}-I)\left(P^{N+1} h_{\epsilon}-h_{0}\right)\right|=\left|\int_{0}^{1} \omega_{\epsilon}(x) v(x) d x\right| \leq C \epsilon r_{0}^{N}$

where the constant $C$ is uniform in $\epsilon$ small. This estimates the second term of (7).

Observe also from (8) that for $N=o\left(\log \epsilon^{-1}\right)$

$$
\left|\lambda^{-N}-1\right|=\mathcal{O}(1) N \epsilon
$$

which implies

$$
\left|\left(\lambda^{-N}-1\right) e_{0}\left((Q-P) h_{0}\right)\right| \leq \mathcal{O}(1) \epsilon^{2} N .
$$

Using perturbation theory with $\theta=\theta_{0}$ we have for $\epsilon$ small enough

$$
h_{\epsilon}=\lambda(\epsilon)^{-N} Q^{N} 1+\lambda(\epsilon)^{-N} u
$$

with

$$
\vee u+\|u\|_{1} \leq \theta_{0}^{-1} \mid\|u\| \|_{\theta_{0}} \leq K r_{1}^{N},
$$

where $r_{1}=2^{-1 / n_{1}}$ and $K$ is a constant independent of $\epsilon$ small.

We have as above

$$
\begin{aligned}
& \lambda(\epsilon)^{-N}\left|\sum_{j=0}^{N-1} \lambda^{-j-1} e_{0}\left((Q-P) P^{j}(Q-P) u\right)\right| \\
& =\lambda(\epsilon)^{-N}\left|\sum_{j=0}^{N-1} \lambda^{-j-1} e_{0}\left((\tilde{Q}-I) P^{j+1}(Q-P) u\right)\right| \\
& \leq \lambda(\epsilon)^{-N} \sum_{j=0}^{N-1} \lambda^{-j-1}\left\|\omega_{\epsilon}\right\|_{1}\left\|P^{j+1}(Q-P) u\right\|_{\infty} \leq \mathcal{O}(1) \epsilon N r_{1}^{N},
\end{aligned}
$$

where for the last inequality we use (LY), Proposition 3, Lemma 6 and (9). 
We finally have to estimate for $j>0$ the quantity

$$
e_{0}\left((Q-P) P^{j}(Q-P) Q^{N} 1\right) \text {. }
$$

Note that

$$
e_{0}\left((Q-P) P^{j}(Q-P) Q^{N} 1\right)=e_{0}\left((\tilde{Q}-I) P^{j+1}(\tilde{Q}-I) P Q^{N} 1\right) .
$$

Using Lemma 6 and the elementary properties of $P$, we have

$$
e_{0}\left((\tilde{Q}-I) P^{j+1}(\tilde{Q}-I) P Q^{N} 1\right)=-\int_{0}^{1} \omega_{\epsilon}\left(f^{j+1}(x)\right)\left((\tilde{Q}-I) P Q^{N} 1\right)(x) d x .
$$

The idea now is to locate the support of $\omega_{\epsilon} \circ f^{j+1}$. We define the sequence of numbers $\delta(n)$ for $n>0$ by

$$
\delta(n)=\inf _{0 \leq s \leq n} d\left(f^{-s-1}(\{0,1\}), \bigcup_{j=1}^{n} f^{j}(\partial \mathcal{A})\right) .
$$

Note that from hypothesis $\mathbf{H 3}$ we have for any $n>0 \delta(n)>0$. We denote by $\eta$ the number

$$
\eta=\inf _{x, n}\left|\left(f^{n}\right)^{\prime}(x)\right|
$$

Note that from the expansivity it follows that $\eta>0$. Note also that by definition $\delta(n)$ is decreasing in $n$. We now claim that if $\delta(s+1)>\epsilon / \eta$ and $\epsilon$ is small enough, the support of $\omega_{\epsilon} \circ f^{s+1}$ is contained in

$$
\left\{x: d\left(x, f^{-s-1}(\{0,1\})\right)<\epsilon / \eta\right\} .
$$

The proof is again recursive, the case $s=0$ is from Lemma 6 . We now observe that if $a$ is a point such that $f^{s+1}(a) \in\{0,1\}$, and $\delta(s+2)>\epsilon / \eta$, then the interval $J$ of width $\epsilon / \eta$ around $a$ does not meet $f(\partial \mathcal{A})$. Therefore the preimages of $J$ are well defined and the induction follows. We now have

$$
\begin{aligned}
e_{0}\left((\tilde{Q}-I) P^{j+1}(\tilde{Q}-I) P Q^{N} 1\right) & \\
=- & \sum_{a \in f^{-j-1}(\{0,1\})} \int_{a-\epsilon / \eta}^{a+\epsilon / \eta} \omega_{\epsilon} \circ f^{j+1}(x)(\tilde{Q}-I) P Q^{N} 1(x) d x .
\end{aligned}
$$

We now impose $\delta(N)>\epsilon / \eta$, and also

$$
\epsilon \sigma^{N}+\frac{\epsilon}{\eta} \leq \delta(N) / 2
$$

Note that when $\epsilon$ tends to zero we can also assume that $N$ tends to infinity with $N \leq o\left(\log \epsilon^{-1}\right)$. This implies for $0 \leq s \leq N$

$$
\left\{x: d\left(x, \bigcup_{j=1}^{N} f^{j}(\partial \mathcal{A})\right) \leq \epsilon \sigma^{N}\right\} \cap \operatorname{supp} \omega_{\epsilon} \circ f^{s+1}=\emptyset .
$$

Observe that if $g$ is differentiable on an interval $[a, b] \subset[\epsilon, 1-\epsilon]$ and such that $\left|g_{[a, b]}^{\prime}\right| \leq R$, then

$$
\sup _{x \in[a+\epsilon, b-\epsilon]}|\tilde{Q} g(x)-g(x)| \leq R \epsilon .
$$


Therefore we get using Corollary 8 and $N=o\left(\log \epsilon^{-1}\right)$

$$
\left|\sum_{j=0}^{N-1} \lambda^{-j-1} e_{0}\left((Q-P) P^{j}(Q-P) Q^{N} 1\right)\right| \leq N \epsilon^{2} K^{N} l^{N} / \eta .
$$

Grouping together all the previous estimates we get the estimate on $\lambda(\epsilon)$. We have also for any function $u$ of bounded variation

$$
\mathbb{E}_{h_{\epsilon} d x}\left(u\left(X_{1}\right) \mid X_{1} \in[0,1]\right)=\frac{\int_{0}^{1} u(x) Q h_{\epsilon}(x) d x}{\int_{0}^{1} Q h_{\epsilon}(x) d x}=\int_{0}^{1} u(x) h_{\epsilon}(x) d x,
$$

which means that the measure $h_{\epsilon}(x) d x$ is quasi-invariant.

In order to prove the last part of Theorem 1, note that for $n>k$ the Markov property implies

$$
\begin{aligned}
\mathbb{P}_{x_{0}}\left\{X_{1} \in d x_{1}, \cdots, X_{k} \in d x_{k} \mid \tau>n\right\} & \\
& =\frac{\mathbb{P}_{x_{k}}\{\tau>n-k\}}{\mathbb{P}_{x_{0}}\{\tau>n\}} \mathbb{P}_{x_{0}}\left\{X_{1} \in d x_{1}, \cdots, X_{k} \in d x_{k}\right\} \\
& =\prod_{l=0}^{k-1} \frac{\mathbb{P}_{x_{l+1}}\{\tau>n-l-1\}}{\mathbb{P}_{x_{l}}\{\tau>n-l\}} \mathbb{P}_{x_{l}}\left\{X_{1} \in d x_{l+1}\right\} .
\end{aligned}
$$

We have also

$$
\mathbb{P}_{x}\left\{X_{1} \in d y\right\}=\varphi\left\{\frac{y-f(x)}{\epsilon}\right\} \frac{d y}{\epsilon} .
$$

On the other hand, from the spectral decomposition

$$
Q^{n}=h_{\epsilon} \lambda(\epsilon)^{n} e_{\epsilon}+T^{n}
$$

and the bounds on $T^{n}$ we get

$$
\begin{aligned}
\frac{\mathbb{P}_{y}\{\tau>n-1\}}{\mathbb{P}_{x}\{\tau>n\}} & =\frac{Q^{n-1} 1(y)}{Q^{n} 1(x)}=\frac{h_{\epsilon}(y) \lambda(\epsilon)^{n-1} e_{\epsilon}(1)+T^{n-1} 1(y)}{h_{\epsilon}(x) \lambda(\epsilon)^{n} e_{\epsilon}(1)+T^{n} 1(x)} \\
& \underset{n \rightarrow \infty}{\longrightarrow} \lambda(\epsilon)^{-1} \frac{h_{\epsilon}(y)}{h_{\epsilon}(x)} .
\end{aligned}
$$

It follows now at once from the above expressions that the limit law

$$
\lim _{n \rightarrow \infty} \mathbb{P}_{x_{0}}\left\{X_{1} \in d x_{1}, \cdots, X_{k} \in d x_{k} \mid \tau>n\right\}
$$

exists and defines a Markovian process with transition kernel

$$
M(x, d y)=\lambda(\epsilon)^{-1} \frac{h_{\epsilon}(y)}{h_{\epsilon}(x)} \varphi\left(\frac{y-f(x)}{\epsilon}\right) \frac{d y}{\epsilon} .
$$

Proof of Theorem 2. We first observe that the operator $\tilde{Q}$ has a kernel $\epsilon^{-1} \varphi((x-y) / \epsilon)$. It follows easily since $\varphi$ is $C^{1}$ that this operator is compact in $\mathcal{B}$. It is also compact (in fact Hilbert Schmidt) in $L^{2}$ and maps this space in $\mathcal{B}$. Therefore, the spectrum in $\mathcal{B}$ and in $L^{2}$ coincide. Moreover, since $\varphi$ is symmetric $\tilde{Q}$ is a self adjoint operator in $L^{2}$ which maps positive functions to positive functions. It is also obvious that there is a large iterate which is positivity improving. Therefore, by the Krein-Rutmann theorem ([KR]) the peripheral spectrum is composed 
of a simple positive eigenvalue. The result follows at once from a trivial application of the minimax principle using the trial function $\sin (\pi x)[\mathrm{K}, \mathrm{I} . \S 10]$.

\section{Analysis of a Deterministic PeRturbation}

We consider the simple map $f_{0}(x)=2 x(\bmod 1)$. We then choose a (small) positive number $1 / 2>\epsilon>0$ and define the interval $K=[1-\epsilon, 1]$ and the discontinuous map $f_{\epsilon}$ by

$$
f_{\epsilon}(x)= \begin{cases}f_{0}(x) & \text { if } 0 \leq x<1-\epsilon \\ 1-2|x-1+\epsilon / 2| & \text { if } 1-\epsilon \leq x \leq 1 .\end{cases}
$$

The map $f_{\epsilon}$ has the interval $K$ as an invariant segment and one can show that for Lebesgue almost any initial condition, the trajectory will end in $K$ (this will also be a consequence of the results below). As we will see below, $f_{\epsilon}$ has a unique a.c.i.m ergodic and mixing with support in $K$ (and we have the SRB property with respect to the Lebesgue measure of the interval $[0,1])$. Therefore, if we let our parameter $\epsilon$ tend to zero the SRB measure of $f_{\epsilon}$ converges to the Dirac measure at the point 1 which is not the SRB measure of $f_{0}$ (the Lebesgue measure). This example is a cooked up version of an example of Keller [Ke] where the perturbed map develops an invariant segment. We conjecture however that the analysis developed below is far more general and could be extended to perturbations of maps which have a periodic point in the boundary of their defining partition.

Let $P_{0}$ and $P_{\epsilon}$ denote the transfer operators of the maps $f_{0}$ and $f_{\epsilon}$ respectively. We will be interested in estimating the spectrum of $P_{\epsilon}$ and compare it to the spectrum of $P_{0}$ for $\epsilon$ small. Our main result is as follows, using the notation $\sigma(\cdot)$ for the spectrum of an operator, and $D_{r}$ for a disk of radius $r$ in the complex plane centered at the origin.

Theorem 9. There is a number $1>r>0$ such that in the space of functions of bounded variation

i) $\sigma\left(P_{0}\right)=\{1\} \cup \Sigma_{0}$ with $\Sigma_{0} \subset D_{r}$. Moreover, 1 is a simple eigenvalue with the eigenvector 1 , and the eigenform $e_{0}$ is given by the integration over the Lebesgue measure.

ii) There is a number $\epsilon_{0}>0$ such that if $\left.\left.\epsilon \in\right] 0, \epsilon_{0}\right]$, there is a number $1>\lambda(\epsilon)>$ $1-o\left(1 / \log \epsilon^{-1}\right)$ such that

$$
\sigma\left(P_{\epsilon}\right)=\{1, \lambda(\epsilon)\} \cup \Sigma_{\epsilon} \quad \text { with } \quad \Sigma_{\epsilon} \subset D_{r} .
$$

Moreover, 1 and $\lambda(\epsilon)$ are simple eigenvalues, with eigenvectors $\chi_{K}$ and $h_{K}$ respectively. $h_{K}$ is a non negative function which converges to 1 (in the $L^{1}$ norm) if $\epsilon \rightarrow 0$. The measure $h_{K} d x$ is a quasi-invariant measure for $f_{\epsilon}$ on $K^{c}$. The eigenform $\mu_{K}$ is a probability measure, and the measure $h_{K} d \mu_{K}$ is an invariant measure for $f_{\epsilon}$ which converges weakly to the Lebesgue measure when $\epsilon \rightarrow 0$.

So we see that in this case the problem of the non convergence of the perturbation is resolved in the following way. The SRB measure for the map $f_{\epsilon}$ converges to the Dirac measure at the point 1 . There is however another measure, corresponding to an eigenvalue near 1 but smaller than one which does converge to the SRB measure of $f_{0}$. Technically, when looking at the Lasota and Yorke estimate for the transfer operator $P_{\epsilon}$, one gets large constants because of the presence of the small invariant segment. It is however natural in the presence of an invariant segment to worry 
about the trajectories which will never enter that segment (or enter only after a very long time). These trajectories are asymptotically distributed according to an invariant measure which is supported by an invariant Cantor set. This measure was essentially constructed in [CG], but for the convenience of the reader we will repeat the proof in the present context (which turns out to be slightly simpler). It turns out that the number $-1 / \log \lambda(\epsilon)$ is exactly the (exponential) escape rate from the interval $K^{c}$ (the complement of $K$ ), and $h_{K} d \mu_{K}$ is an invariant measure for the dynamics on the invariant Cantor set.

The assertion i) of the above theorem is of course easy and well known, we have repeated it only for completeness of the result.

Below we will denote by $Q$ the operator

$$
Q=P_{\epsilon} \chi_{K}
$$

Note that since $K$ is invariant we have $Q=\chi_{K} P_{\epsilon} \chi_{K}$. This operator maps functions on $[0,1]$ into functions with support in $K$. Moreover, if one considers the functions with support in $K$, it is the transfer operator of $\left.f_{\epsilon}\right|_{K}$. So we have also the following easy and well known result.

Proposition 10. On the Banach space of functions of bounded variation with support in $K$, we have for some number $0<r_{1}<1$ independent of $K$

$$
\sigma(Q)=\{1\} \cup \Theta \quad \text { with } \quad \Theta \subset D_{r_{1}} .
$$

The eigenvector associated to 1 is the function $\chi_{K}$, the associated linear form is the integration with respect to the measure $\chi_{K}(x) d x$.

The following result is obtained by an easy explicit computation using mainly that $\chi_{K^{c}} P_{\epsilon} \chi_{K}=0$ since $K$ is an invariant segment. Denoting by $S$ the operator $P_{\epsilon} \chi_{K^{c}}$, we have $P_{\epsilon}=S+Q$ and $S Q=0$.

Lemma 11. If $\zeta$ is a complex number, with modulus larger than

$$
\max \left(\left\|P_{\epsilon}\right\|,\|Q\|,\|S\|\right),
$$

then

$$
\left(\zeta-P_{\epsilon}\right)^{-1}=\left(\chi_{K^{c}}+\zeta \chi_{K}(\zeta-Q)^{-1} \chi_{K}\right)(\zeta-S)^{-1}
$$

Proof. If $|\zeta|$ is larger than $\max \left(\left\|P_{\epsilon}\right\|,\|Q\|,\|S\|\right)$ we can use Neuman's series to compute the resolvents of the operators. It is thus enough to prove that

$$
\zeta-S=\left(\zeta-P_{\epsilon}\right)\left(\chi_{K^{c}}+\zeta \chi_{K}(\zeta-Q)^{-1} \chi_{K}\right) \text {. }
$$

Expanding the right hand side, and using $P_{\epsilon} \chi_{K}=Q$ we get

$$
\begin{gathered}
\left(\zeta-P_{\epsilon}\right)\left(\chi_{K^{c}}+\zeta \chi_{K}(\zeta-Q)^{-1} \chi_{K}\right) \\
=\zeta \chi_{K^{c}}-S+\sum_{n=0}^{\infty} \zeta^{-n+1} Q^{n} \chi_{K}-\sum_{n=0}^{\infty} \zeta^{-n} Q^{n+1} \chi_{K}=\zeta \chi_{K^{c}}-S+\zeta \chi_{K} .
\end{gathered}
$$

Note that this result says that due to the presence of the invariant segment, we have succeeded in bloc triangularizing the operator $P_{\epsilon}$. Let $\mathcal{R}(A)$ denote the resolvent set of the operator $A$, and for a compact subset $B$ of the complex plane let 
$B^{c}$ denote its complement and $B^{a c \infty}$ its arcwise connected component of infinity. By analytic continuation, we get the following result.

\section{Proposition 12.}

$$
\sigma\left(P_{\epsilon}\right) \subset\left(\mathcal{R}(Q)^{a c \infty}\right)^{c} \cup\left(\mathcal{R}(S)^{a c \infty}\right)^{c} .
$$

Moreover, if

$$
\lambda \in\left(\sigma_{p}(S) \cap \overline{\mathcal{R}(Q)^{a c \infty}}\right) \Delta\left(\sigma_{p}(Q) \cap \overline{\mathcal{R}(S)^{a c \infty}}\right),
$$

then $\lambda \in \sigma_{p}\left(P_{\epsilon}\right)$ (where $\Delta$ denotes the symmetric difference between sets).

Proof. The proof of the inclusion of the spectra is immediate from the previous Lemma since by analytic continuation we have

$$
\left(\mathcal{R}(Q)^{a c \infty}\right) \cap\left(\mathcal{R}(S)^{a c \infty}\right) \subset \mathcal{R}\left(P_{\epsilon}\right) .
$$

The second part follows from elementary properties of meromorphic functions.

We now determine the spectrum of $S$. As mentioned before, this is a simple application of the techniques developed in [CG]. We repeat the details here for the convenience of the reader. The major observation is that $S=P_{\epsilon} \chi_{K^{c}}=P_{0} \chi_{K^{c}}$ and we are going to consider $S$ as a perturbation of $P_{0}$. This does not work immediately since with the standard norm, the operator $S-P_{0}$ has a norm of order 1 , and therefore $S$ does not look as a small perturbation of $P_{0}$. In order to cope with this problem, we will use the same technique of balancing norms as in section III.

Theorem 13. There is a number $1>r_{2}>0$ and a number $\epsilon_{0}>0$ such that if $\left.\epsilon \in] 0, \epsilon_{0}\right]$, there is a number $1>\lambda(\epsilon)>1-1 / \log \epsilon^{-1}$ such that in the space of functions of bounded variation

$$
\sigma(S)=\{\lambda(\epsilon)\} \cup \Omega_{\epsilon} \quad \text { with } \quad \Omega_{\epsilon} \subset D_{r_{2}} .
$$

Moreover, $\lambda(\epsilon)$ is a simple eigenvalue, with eigenvector denoted by $h_{K} . h_{K}$ is a non negative function which converges to 1 if $\epsilon \rightarrow 0$. The measure $h_{K} d x$ is quasi invariant. The eigenform $\mu_{K}$ is a positive measure, the measure $h_{K} d \mu_{K}$ is an invariant measure and $\mu_{K}$ converges weakly to the Lebesgue measure if $\epsilon \rightarrow 0$.

Proof. As explained above, we are going to apply perturbation theory in a Banach space with an equivalent norm. Let first $q$ be a positive number large enough, to be fixed later on independently of $\epsilon$. We denote by $\Delta_{q}$ the operator

$$
\Delta_{q}=S^{q}-P_{0}^{q} .
$$

We first observe that for $m>1$ we have

$$
S^{m}=P_{0}^{m}-\sum_{l=0}^{m-1} S^{m-1-l} P_{0} \chi_{K} P_{0}^{l}=P_{0}^{m}-\sum_{l=0}^{m-1} P_{0}^{m-l} \chi_{K} S^{l} .
$$

Combining these two expressions, we obtain easily

$$
S^{q}=P_{0}^{q}-\sum_{j=0}^{q-1} P_{0}^{q-j} \chi_{K} P_{0}^{j}+\sum_{j=0}^{q-1} \sum_{l=0}^{j-1} P_{0}^{q-j} \chi_{K} S^{j-1-l} P_{0} \chi_{K} P_{0}^{l} .
$$

Let now $q_{K}$ be the largest integer such that for any $0<j<q_{K}$ we have

$$
f_{0}^{j}\left(f_{0}(K) \cap K^{c}\right) \cap K=\emptyset .
$$


Note that $q_{K}$ tends to infinity if $\epsilon$ goes to zero - in fact with our example

$$
\left|\log \epsilon^{-1} / \log 2-q_{K}\right| \leq \mathcal{O}(1),
$$

and if $0<i<q_{K}$ we have

$$
\chi_{K} S^{i} P_{0} \chi_{K}=0
$$

Therefore, if $q \leq q_{K}$ we have

$$
\Delta_{q}=-\sum_{j=0}^{q-1} P_{0}^{q-j} \chi_{K} P_{0}^{j}+\sum_{j=0}^{q-1} P_{0}^{q-j} \chi_{K} P_{0} \chi_{K} P_{0}^{j-1} .
$$

From now on we will always assume that $q \leq q_{K}$.

We see that due to the special geometry, although $\Delta_{q}$ is a priori a sum of $2^{q}-1$ elements, it turns out to be only a sum of $2 q$ terms. As we will see below, going from an exponential estimate in $q$ to a polynomial estimate is the key to a successful bound. We now estimate separately the norm of each term in $\Delta_{q}$. We first recall the Lasota-Yorke estimate, namely there is a constant $C>1$, a number $0<\alpha<1$ and a positive number $\Gamma>1$ such that for any function $g$ of bounded variation and for any integer $n$

$$
\bigvee\left(P_{0}^{n} g\right) \leq C \alpha^{n} \vee g+\Gamma\|g\|
$$

where $\|\cdot\|$ denotes the $L^{1}$ norm with respect to the normalized Lebesgue measure of the interval $[0,1]$. We also recall that $\left\|P_{0} g\right\| \leq\|g\|$. We now have for $0 \leq j \leq q-1$ and for any $g$ of bounded variation

$$
\begin{aligned}
& \bigvee\left(P_{0}^{j} \chi_{K} P_{0}^{q-j} g\right) \leq C \alpha^{j} \bigvee\left(\chi_{K} P_{0}^{q-j} g\right)+\Gamma\left\|\chi_{K} P_{0}^{q-j} g\right\| \\
& \leq C \alpha^{j} \bigvee\left(P_{0}^{q-j} g\right)+2 C \alpha^{j}\left\|P_{0}^{q-j} g\right\|_{\infty}+\epsilon \Gamma\left\|P_{0}^{q-j} g\right\|_{\infty},
\end{aligned}
$$

where $\|\cdot\|_{\infty}$ is the $L^{\infty}$ norm, and we have used the simple estimate

$$
\vee\left(g_{1} g_{2}\right) \leq \vee\left(g_{1}\right)\left\|g_{2}\right\|_{\infty}+\vee\left(g_{2}\right)\left\|g_{1}\right\|_{\infty},
$$

together with $\vee \chi_{K}=2$.

We now continue using the Lasota-Yorke estimate and we get using for a function $u$ of bounded variation $\|u\|_{\infty} \leq \vee(u)+\|u\|$ and $\left\|P_{0} u\right\| \leq\|u\|$

$$
\bigvee\left(P_{0}^{j} \chi_{K} P_{0}^{q-j} g\right) \leq \mathcal{O}(1)\left(\alpha^{q-1}+\epsilon \alpha^{q-j}\right) \vee(g)+\mathcal{O}(1)\left(\alpha^{j}+\epsilon\right)\|g\| .
$$

A similar estimate can be obtained for the second term of $\Delta_{q}$, either directly or by simply observing that for $\epsilon$ small we have

$$
\chi_{K} P_{0} \chi_{K}=P_{0} \chi_{\tilde{K}}
$$

where $\tilde{K}$ is also an interval. From this it follows that

$$
\bigvee\left(\Delta_{q} g\right) \leq A\left(q \alpha^{q}+\epsilon\right) \vee(g)+A(1+q \epsilon)\|g\|,
$$

where $A$ is a positive constant (independent of $q, g$ and $\epsilon$ ). We have also

$$
\left\|P_{0}^{j} \chi_{K} P_{0}^{q-j} g\right\| \leq\left\|\chi_{K} P_{0}^{q-j} g\right\| \leq \epsilon\left\|P_{0}^{q-j} g\right\|_{\infty} \leq \epsilon\left(C \alpha^{q-j} \vee(g)+\Gamma\|g\|\right),
$$

which implies immediately (with the similar estimate for the second term of $\Delta_{q}$ )

$$
\left\|\Delta_{q} g\right\| \leq \epsilon B(\vee(g)+q\|g\|)
$$


where $B$ is a positive constant (independent of $q, g$ and $\epsilon$ ). The main observation is now that for a fixed (large) $q$, if we take $\epsilon$ small enough, only the coefficient of $\|g\|$ in (11) is of order one, all the other coefficients are small. This suggests the introduction of a balanced norm depending on a parameter $\theta>0$ given by

$$
\|\| g\left\|_{\theta}=\theta \vee(g)+\right\| g \| \text {. }
$$

Note that all these norms are equivalent and we have for $\theta^{\prime}>\theta$

$$
\left\|\left|g\left\|_{\theta} \leq\right\|\right| g\right\|_{\theta^{\prime}} \leq \frac{\theta^{\prime}}{\theta}\|g\|_{\theta} .
$$

We now apply the spectral decomposition theorem to $P_{0}$ (see $[\mathrm{K}]$ ). We conclude that for any number $\xi \in] r_{1}, 1[$, there is a constant $\Psi$ which depends only on $\xi$ (and which can be assumed larger than one), there is a projection operator $\pi_{0}$ of rank one and an operator $R$ satisfying $\pi_{0} R=R \pi_{0}=0$ and such that

$$
P_{0}=\pi_{0}+R \quad \text { and for any integer } n \quad\left\|R^{n}\right\|_{1} \leq \Psi \xi^{n} .
$$

In other words, $\pi_{0}$ is the spectral part of $P_{0}$ corresponding to the eigenvalue 1 , and $R$ is the rest in the spectral decomposition. Note that $\pi_{0}$ is a rank one operator consisting of a linear form $F$ which is the integration on $[0,1]$ with the Lebesgue measure and an eigenvector which is the constant function one. Therefore, we have by direct computations for any $\theta>0,\||| F \mid\|_{\theta}=1$ and $\||1|\| \|_{\theta}=1$ which imply \|\|$\pi_{0} \|_{\theta}=1$. For the operator $R$, on the other hand, we have only the trivial estimate

$$
\left\|R^{n}\right\|_{\theta} \leq \Psi \theta^{-1} \xi^{n}
$$

for any integer $n$ and any $\theta>0$.

We are now finally in a position to choose the number $q$. We have of course

$$
S^{q}=\pi_{0}+R^{q}+\Delta_{q}
$$

Using our above estimates we conclude that for any $\theta>0$, provided $q \leq q_{K}$ we have

$$
\left\|R^{q}+\Delta_{q}\right\|_{\theta} \leq \theta^{-1} \Psi \xi^{q}+A\left(q \alpha^{q}+\epsilon\right)+A \theta(1+q \epsilon)+\epsilon B \theta^{-1}+q \epsilon B .
$$

Now we see that if we could take $\theta$ small, the term of order one coming from (11) will be small in the new norm. In order not to upset too much the bound on the first term, we define an optimal $\theta$ by

$$
\theta=\theta(q)=\sqrt{\frac{\Psi \xi^{q}}{A}}
$$

There are now several possible choices for $q$. A first natural one will be such that the right hand side of (12) will be small for $\epsilon$ small enough. Namely, one can take for example the smallest $q$ such that

$$
2 \sqrt{A \Psi \xi^{q}}+A q \alpha^{q} \leq \frac{1}{50} \text { and } \theta(q)<1 .
$$

Denote this number by $q_{1}$. Once this $q=q_{1}$ has been chosen (and the corresponding $\theta_{1}=\theta\left(q_{1}\right)$ set as above), we have

$$
\|\| R^{q}+\Delta_{q} \|_{\theta_{1}} \leq \frac{1}{50}+\epsilon\left(A+A \theta\left(q_{1}\right) q_{1}+B \theta\left(q_{1}\right)^{-1}+B q_{1}\right) .
$$


Now we choose $\epsilon_{0}$ by

$$
\epsilon_{0}=\frac{1}{50\left(A+A \theta\left(q_{1}\right) q_{1}+B \theta\left(q_{1}\right)^{-1}+B q_{1}\right)} .
$$

With this choice, we can apply perturbation theory (using $\left\|R^{q}+\Delta_{q}\right\| \|_{\theta}<1 / 24$, see [K.II. §3] and $\left\|\mid \pi_{0}\right\|_{\theta}=1$ ) as in the proof of Proposition 5 to ensure that outside $D_{1 / 2}$ the spectrum of $S^{q_{1}}$ consists of a unique simple eigenvalue $\lambda(\epsilon)$. For the eigenvector $h_{K}$ we have

$$
\left\|\mid h_{0}-h_{K}\right\|_{\theta} \leq \mathcal{O}(1)
$$

which by the equivalence of norms (note that $q_{1}$ and hence $\theta\left(q_{1}\right)$ do not depend on $\epsilon)$ implies

$$
\vee h_{K} \leq \mathcal{O}(1) \quad \text { and if }\left\|h_{0}\right\|=1, \quad\left\|h_{K}\right\| \leq \mathcal{O}(1),
$$

uniformly in $\epsilon<\epsilon_{0}$.

A choice of $q$ leading to a finer estimate is the number $q_{2}=\beta \log \epsilon^{-1}$ where $\beta=$ $\inf \left(1 / \log 2,1 / \log \alpha^{-1}, 1 / \log \xi^{-1}\right) / 2$, where $\beta<\frac{1}{2} \log 2$ is to ensure condition (10). Applying again perturbation theory with this choice of $q_{2}$ (and the corresponding $\theta_{2}=\theta\left(q_{2}\right)$ ), we conclude using (12) that for $\epsilon$ small enough, if $g$ is a function of bounded variation

$$
\left|\mu_{K}(g)-e_{0}(g)\right| \leq \mathcal{O}(1) \epsilon^{\gamma} \log \epsilon^{-1}|||g| \|_{\theta_{2}}
$$

where

$$
\gamma=\inf \left(\beta \log \xi^{-1} / 2,1-\beta \log \xi^{-1} / 2, \beta \log \alpha^{-1}\right) .
$$

By definition this implies

$$
\left|\mu_{K}(g)-e_{0}(g)\right| \leq \mathcal{O}(1) \epsilon^{\gamma} \log \epsilon^{-1}|||g| \| \mid .
$$

We also have from perturbation theory as in the proof of Proposition 5

$$
\left.\left\|\left|h_{K}-1\right|\right\|\right|_{\theta_{2}} \leq \mathcal{O}(1) \epsilon^{\gamma} \log \epsilon^{-1},
$$

hence from the definition of the $\theta$ norm

$$
\left\|h_{K}-1\right\| \leq \mathcal{O}(1) \epsilon^{\gamma} \log \epsilon^{-1} .
$$

As another consequence of perturbation theory, we have of course

$$
|\lambda(\epsilon)-1| \leq \mathcal{O}(1) \epsilon^{\gamma} \log \epsilon^{-1},
$$

which implies $\lambda(\epsilon) \rightarrow 1$ when $\epsilon \rightarrow 0$.

We also observe that $\mu_{K}$ is positive on positive functions, therefore by density it extends to a positive functional on continuous functions and is therefore a positive measure (which can be normalized to a probability measure). It is easy to verify that it is supported by the invariant Cantor set

$$
\bigcap_{n=0}^{\infty} f_{\epsilon}^{-n}\left(K^{c}\right)
$$

i.e. $\mu_{K}$ is supported by the Cantor set of trajectories which never leave $K^{c}$.

From the above estimate, we also conclude that $\mu_{K}$ converges weakly when $\epsilon \rightarrow 0$ to the SRB measure of $f_{0}$ which is the Lebesgue measure.

If $u$ is a function of bounded variation, we have by definition 


$$
\begin{gathered}
\int \prod_{0}^{n} \chi_{K^{c}} \circ f_{\epsilon}^{j}(x) h_{K}(x) u(x) d x=\int \chi_{K^{c}}(x) S^{n-1}\left(h_{K} u\right)(x) d x \\
=\lambda(\epsilon)^{n-1}\left(\int_{K^{c}} h_{K}(x) d x\right) \mu_{K}\left(\chi_{K^{c}} h_{K} u\right)+o\left(\lambda(\epsilon)^{n-1}\right) .
\end{gathered}
$$

From the identity

$$
S\left(u \circ f_{\epsilon} \chi_{K^{c}} h_{K}\right)=P_{\epsilon}\left(u \circ f_{\epsilon} \chi_{K^{c}} h_{K}\right)=u P_{\epsilon}\left(\chi_{K^{c}} h_{K}\right)=u S\left(h_{K}\right)=\lambda(\epsilon) u h_{K}
$$

we have

$$
\begin{gathered}
\int \prod_{0}^{n} \chi_{K^{c}} \circ f_{\epsilon}^{j}(x) u \circ f_{\epsilon}(x) h_{K}(x) d x=\int \prod_{0}^{n-1} \chi_{K^{c}} \circ f_{\epsilon}^{j}(x) u(x) S\left(h_{K}\right)(x) d x \\
=\lambda(\epsilon)^{n-1}\left(\int_{K^{c}} h_{K}(x) d x\right) \mu_{K}\left(h_{K} u \chi_{K^{c}}\right)+o\left(\lambda(\epsilon)^{n-2}\right) .
\end{gathered}
$$

Since the support of $\mu_{K}$ is contained in the interior of $K^{c}$, we derive by letting $n$ tend to infinity that

$$
\mu_{K}\left(h_{K} u \circ f_{\epsilon}\right)=\mu_{K}\left(h_{K} u\right),
$$

which is the statement of the invariance of $h_{K} d \mu_{K}$ for $f_{\epsilon}$.

We have also for a function $u$ of bounded variation

$$
\begin{aligned}
\frac{\int u \circ f_{\epsilon}(x) \chi_{K^{c}}(x) h_{K}(x) d x}{\int 1 \circ f_{\epsilon}(x) \chi_{K^{c}}(x) h_{K}(x) d x} & =\frac{\int u(x) S h_{K}(x) d x}{\int S h_{K}(x) d x} \\
& =\frac{\lambda(\epsilon) \int u(x) h_{K}(x) d x}{\lambda(\epsilon) \int h_{K}(x) d x}=\int u(x) h_{K}(x) d x
\end{aligned}
$$

since $h_{K}$ has been normalized to have integral 1 . This implies that the measure $h_{K} d x$ (with support on $K^{c}$ ) is quasi invariant.

Theorem 9 is now an immediate consequence of Theorem 13 and Proposition 12.

\section{The Markov Case}

This is a simple but instructive exercise. Choose $\epsilon=2^{-n}$, then it is easy to verify that the map $f$ is Markov. In fact the Markov partition is made up of $n+2$ atoms given by

$$
A_{j}= \begin{cases}{\left[1-2^{-j}, 1-2^{-j-1}\right]} & \text { for } 0 \leq j \leq n, \\ {\left[1-2^{-n-1}, 1\right]} & \text { for } j=n+1 .\end{cases}
$$

If $h$ is a function which belongs to the $\sigma$-algebra determined by the above Markov partition, we denote by $h_{j}$ its value on the atom $A_{j}$. The transfer operator acting on $h$ is easily seen to be given by

$$
\left(P_{\epsilon} h\right)_{j}= \begin{cases}\left(h_{0}+h_{j+1}\right) / 2 & \text { for } 0 \leq j \leq n-2, \\ h_{0} / 2 & \text { for } j=n-1, \\ \left(h_{0}+h_{n}+h_{n+1}\right) / 2 & \text { for } j=n \\ \left(h_{0}+h_{n}+h_{n+1}\right) / 2 & \text { for } j=n+1 .\end{cases}
$$


It follows easily from this explicit expression that the two dimensional linear subspace $h_{0}=\cdots=h_{n-1}=0$ is invariant, and in this subspace, the spectrum consists of a simple eigenvalue 1 with eigenvector $h_{n}=h_{n+1}=1$ and an eigenvalue 0 with eigenvector $h_{n}=-h_{n+1}=1$. Moreover, the matrix for $P_{\epsilon}$ is triangular, and one gets easily the eigenvalue equation for the rest of the spectrum which is given (except for the spurious root $\lambda(\epsilon)=1 / 2$ ) by

$$
2^{n+1} \lambda(\epsilon)^{n}(\lambda(\epsilon)-1)+1=0
$$

from which one can easily extract for large $n$ the behavior of the largest eigenvalue which is given by

$$
\lambda(\epsilon)=1-2^{-(n+1)}+\mathcal{O}\left(n 4^{-n}\right) .
$$

It also follows easily that there is no other eigenvalue outside the disk of radius $1 / 2+\mathcal{O}(1) / n$.

\section{ACKNOWLEDGMENTS}

The authors are indebted to program ECOS-CONICYT. S.M. was partially supported by FONDECY T 1940405. P.C. would like to thank for their kind hospitality the Mittag Leffler Institute and the Departamento de Ingeniería Matemática of the Universidad de Chile where part of this work was done. The authors are grateful to an anonymous referee for suggesting many useful improvements of the text.

\section{REFERENCES}

[BK] M. Blank and G. Keller, Stochastic stability versus localization in chaotic dynamical systems, Nonlinearity 10 (1997), 81-107. MR 98a:58101

[BY] V. Baladi and L.-S. Young, On the spectra of randomly perturbed expanding maps, Commun. Math. Phys., vol. 156, 1993, pp. 355-385. MR 94g:58712; Erratum, MR 95k:58125

[BIS] V. Baladi, S. Isola and B. Schmitt, Transfer operator for piecewise affine approximations of interval maps, Ann. Inst. H. Poincaré, Physique Théorique, 62 (1995), 251-266. MR 96i: 58142

[BKS] V. Baladi, A. Kondah and B. Schmitt, Random correlations for small perturbations of expanding maps, Random and Computational Dynamics 4 (1996), 179-204. MR 97e:58139

[BWZ] M. N. Bussac, R. B. White and L. Zuppiroli, Particle and heat transport in a partially stochastic magnetic field, Physics Letters A, 190 (1994), 101-105.

[C] P. Collet, Some Ergodic Properties of Maps of the Interval, In "Dynamical Systems \& Frustrated Systems", R.Bamon, J.-M.Gambaudo and S.Martínez editors, Hermann, Paris, 1996.

[CG] P. Collet and A. Galves, Asymptotic distribution of entrance times for expanding maps of the interval, Dynamical Systems and Applications (R. P. Agarwal, ed.), World Scientific, 1995. MR 97b:58083

[CMSM] P. Collet, S. Martínez and J. San Martín, Asymptotic laws for one dimensional diffusions conditioned to non absorption, Ann. of Prob. 23 (1995), 1300-1314. MR 96i:60083

[FKMP] P. Ferrari, H. Kesten, S. Martínez and P. Picco, Existence of quasi-stationary distributions, A renewal dynamical approach. Ann. of Prob. 23 (1995), 501-521. MR 96c:60059

[FW] M. Freidlin and A. Wentzell, Random perturbations of dynamical systems, Springer, Berlin, Heidelberg, New York, 1984. MR 85a:60064

[HK] F. Hofbauer and G. Keller, Ergodic properties of invariant measures for piecewise monotonic transformations, Math. Z. 180 (1982), 119-140. MR 83h:28028

[K] T. Kato, Perturbation Theory for Linear Operators, Springer, Berlin, Heidelberg, New York, 1966. MR 34:3324

[Ke] G. Keller, Stochastic stability in some chaotic dynamical systems, Mh. Math. 94 (1982), 313-333. MR 84k:58130 
[KR] M. G. Krein and M. A. Rutman, Linear operators leaving invariant a cone in a Banach space, Amer. Math. Soc. Transl. Ser. 1, 10 (1962), 199-225.

[LY] A. Lasota and J. Yorke, On the existence of invariant measures for piecewise monotone transformations, Trans. Amer. Math. Soc. 186 (1973), 481-488. MR 49:538

C.N.R.S., Physique Théorique, Ecole Polytechnique, 91128 Palaiseau Cedex, France

E-mail address: collet@cpht.polytechnique.fr

Universidad de Chile, Facultad de Ciencias Físicas y Matemáticas, Departamento de Ingeniería Matemática, Casilla 170-3 Correo 3, Santiago, Chile

E-mail address: smartine@dim.uchile.cl

Université de Bourgogne, Département de Mathématiques, Faculté de Sciences MiRande, BP-138, 21004 Dijon Cedex, France

E-mail address: schmittb@satie.u-bourgogne.fr 